\title{
Itinerários terapêuticos de pessoas com doenças respiratórias crônicas
}

\author{
Therapeutic itineraries of people with chronic respiratory diseases
}

Itinerarios terapéuticos de personas con enfermedades respiratorias crónicas

Marcio Costa de Souza ${ }^{1 *}$, Juliana Costa dos Santos Borges ${ }^{1,}$ Rafaela Silva Santos ${ }^{1}$, Vitória Cézar Santos Gonçalves Brito ${ }^{1}$, Jairrose Nascimento Souza1 ${ }^{1}$, Rocío Andrea Cornejo Quintana1, Magno Conceição das Merces ${ }^{1}$, Fernanda Warken Rosa Camelier ${ }^{1}$, Aquiles Assunção Camelier ${ }^{1,2}$.

\section{RESUMO}

Objetivo: Compreender os Itinerários Terapêuticos de pacientes com doenças respiratórias crônicas nos diversos níveis de atenção à saúde. Métodos: O estudo trata-se de uma abordagem qualitativa e exploratória, realizada em um distrito sanitário de uma capital na região nordeste do Brasil. A técnica para a produção dos dados foi a entrevista semiestruturada com trabalhadores de saúde e pacientes com doenças respiratórias crônicas, o qual utilizou a análise de conteúdo para a interpretação do material coletado. Resultados: Os principais achados produzidos na pesquisa identificadas foram as consequências de uma comunicação ineficiente nas redes de atenção à saúde, o deslocamento excessivo do usuário no processo do cuidado, ocorrida principalmente pela dificuldade de acesso aos serviços no sistema de saúde local. Conclusão: Há um déficit na comunicação entre as redes de atenção à saúde, o que dificulta a resolutividade do cuidar com um olhar integral, o que produz uma rede fragmentada nos seus diferentes níveis.

Palavras-chave: Assistência integral à saúde, Doenças respiratórias, Serviços de saúde.

\section{ABSTRACT}

Objective: Understand the therapeutic itinerary of patients with chronic respiratory diseases at the various levels of health care. Methods: The study is an exploratory and qualitative approach. The technique for data production was the semi-structured interview with health workers and patients with chronic respiratory diseases, which used content analysis to interpret the collected material. Results: The main findings produced in the research identified were the consequences of inefficient communication in the health care networks, the excessive displacement of the user in the care process, occurred mainly due to the difficulty of accessing services in the local health system. Conclusion: There is a deficit in communication between health care networks, which makes it difficult to solve care with a comprehensive view, which produces a fragmented network at its different levels.

Keywords: Comprehensive health care, Respiratory tract diseases, Health services.

\section{RESUMEN}

Objetivo: Conocer los itinerarios terapéuticos de los pacientes con enfermedades respiratorias crónicas en los diferentes niveles asistenciales. Métodos: El estudio es un abordaje cualitativo y exploratorio, realizado en un distrito de salud de una ciudad capital de la región nordeste de Brasil. La técnica de producción de los datos fue la entrevista semiestructurada con trabajadores de salud y pacientes con enfermedades respiratorias crónicas, que utilizó el análisis de contenido para interpretar el material recolectado. Resultados:

1 Universidade do Estado da Bahia, Salvador - BA.

*E-mail: mcsouzafisio@gmail.com

2 Escola Bahiana de Medicina e Saúde Pública, Salvador - BA. 
Los principales hallazgos producidos en la investigación identificada fueron las consecuencias de la comunicación ineficiente en las Redes de Atención de Salud, el desplazamiento excesivo del usuario en el proceso de atención, que se produjo principalmente por la dificultad de acceso a los servicios en el sistema de salud local. Conclusión: Existe un déficit de comunicación entre las redes asistenciales, lo que dificulta la solución de la atención con una visión integral, lo que produce una red fragmentada en sus diferentes niveles.

Palabras clave: Atención integral de salud, Enfermedades respiratorias, Servicios de salud.

\section{INTRODUÇÃO}

O estudo sobre Itinerário Terapêutico (IT) concede uma forma para compreender todo o processo de adoecimento de uma pessoa, desde o aparecimento dos primeiros sinais/sintomas, em seu diagnóstico, até a maneira pela qual a doença é interpretada pelo enfermo e seus familiares, e sobre como lidam com tal situação.

Pode-se afirmar que o IT passou a ser considerado na elaboração do cuidado sob a luz de um conceito denominado comportamento do enfermo (illness behaviour), termo que surgiu em 1960 através de Mechanic e Volkart (COQUEIRO JM, et al., 2018).

A literatura atual remete a importância que o estudo sobre o IT permite, no que diz respeito às interpretações dos processos, que as pessoas ou grupos sociais optam, analisam e aderem a determinados tratamentos. A análise do IT traz um viés extremamente relevante pautado em identificar de forma dinâmica o processo de construção das histórias individuais que caracterizam dado contexto patológico.

As investigações sobre itinerários terapêuticos, ainda que seja uma linha de raciocínio recente no Brasil, detém de potencial suficiente para subsidiar a forma como a qual os serviços de saúde se organizam e/ou como estes podem aprimorar a forma de organização para iniciar ou dar continuidade ao processo do cuidado (3). (SILVA NEK, et al., 2016, CABRAL ALL, 2011).

Segundo dados do Sistema de Informação Hospitalar (SIH/SUS) do Ministério da Saúde, fora observado que entre os anos de 2003 e 2012 as doenças do aparelho respiratório estavam entre as cinco principais causas de internações hospitalares. Estes dados, que evoluem ao longo dos anos, são alarmantes e questionáveis sobre quando e quais as condições de cuidado ao qual estas pessoas são submetidas e como estas o interpretam (BRASIL, 2018).

Desta forma, o modo como as diversas formas de DRC são negligenciadas predispõe ao agravo da patologia e esta evolução não passa despercebida pela análise do IT, posto que, seus estudos tradicionais denotam da importância em compreender os fatores extra biológicos da morbidade.

O entendimento acerca do comportamento do enfermo traduz as definições populares sobre doença, as crenças muitas vezes não questionadas no modelo biomédico e a percepção sobre os processos terapêuticos (SILVA NEK, et al., 2016).

O subsídio maior da compreensão de um IT é a comunicação, por isso o Ministério da Saúde criou em 2010 as Redes de Atenção à Saúde (RAS) que propõem que todas as informações sobre um enfermo sejam compartilhadas e atualizadas entre os três níveis de atenção: primária, secundária e terciária (ZUIM RCB e TRAJMAN A, 2018). Diante do exposto este trabalho tem como objetivo compreender o Itinerário Terapêutico de pacientes com Doenças Respiratórias Crônicas nos diversos níveis de atenção à saúde.

\section{MÉTODOS}

A pesquisa trata-se de uma abordagem qualitativa, exploratória que foi realizada em quatro Unidades de Saúde da Família (USF) escolhidas de forma intencional. Por ser uma pesquisa de natureza qualitativa, o quantitativo dos sujeitos pesquisados não é uma preocupação, pois o intuito é o aprofundamento e abrangência do fenômeno estudado, portanto, o critério não se baseia nos números, assim, amostragem ideal foi aquela que possibilitou observar as múltiplas dimensões sobre IT de pessoas com DRC. 
A composição da amostra foi escolhida a partir da saturação das respostas, desta forma, em vista as questões colocadas aos entrevistados, contribuíram para a efetivação do objetivo da pesquisa, totalizando 23 entrevistados.

Os participantes do estudo foram formados por trabalhadores de saúde do Hospital Geral Roberto Santos (HGRS), trabalhadores de saúde de algumas Unidades de Saúde da Família (USF) e usuários acometidos por DRC e familiares e/ou cuidadores de pessoas com esta condição de saúde. Quando definimos trabalhadores, pôde ser qualquer pessoa que atua naquele ambiente, independente da formação específica para saúde (médicos, enfermeiros, atendentes, e demais trabalhadores do serviço).

Como critério de inclusão os trabalhadores de saúde foram aqueles que atuam diretamente com as pessoas com DRC na USF e quanto aos usuários, pessoas que sofram ou que cuidem das enfermidades estudadas. Quanto ao critério de exclusão: foram menores de 18 anos e os que não desejaram participar do estudo.

Durante as entrevistas, foi utilizado um gravador (Motorola XT311) como forma de garantir a fidedignidade dos depoimentos, todavia, foi apenas utilizado com a devida autorização do participante. Para a realização da entrevista estruturada, a mesma possuía um roteiro em que se motivou compreender a análise do itinerário terapêutico de pessoas com DRC.

Como técnica para a produção de dados, foi realizada a Análise de Conteúdo adaptado por Minayo MCS (2014). No sentido de sistematizar os dados para uma análise, foram realizados os seguintes passos: ordenação dos dados por meio de um mapeamento de todos os dados obtidos (transcrição de gravações, releitura de material, organização de gravações, dos dados do diário de campo, das fontes documentais).

Por conseguinte, foi realizado a classificação dos dados, quando identificamos os núcleos dos sentidos e posteriormente elaboramos as categorias empíricas. Ao momento da análise final, articulamos os dados empíricos com os referenciais teóricos da pesquisa e documentos, promovendo a relação entre o concreto e o abstrato, o geral e o particular, a teoria e a prática.

Assim, a pesquisa foi iniciada após a concordância dos sujeitos por meio da assinatura do Termo de Consentimento Livre e Esclarecido (TCLE), onde estava exposta a proposta do estudo; objetivos; justificativa; riscos e benefícios, como também, a permissão para a divulgação do conteúdo da gravação, transcrição literal e publicação dos resultados da pesquisa, mantendo, no entanto, a garantia do sigilo, anonimato e privacidade dos sujeitos do estudo, o qual esteve baseado na Resolução 466/12 do Conselho Nacional de saúde sobre as Diretrizes e Normas Regulamentadoras de Pesquisa envolvendo seres humanos (CAAE:61478016.0.0000.0057).

\section{RESULTADOS E DISCUSSÃO}

A pesquisa teve participação de 23 entrevistados, entre usuários portadores de DRC e profissionais de saúde desta mesma área. A construção do estudo se baseou nos relatos sobre itinerário terapêutico, caracterizando a relevância em compreender a trajetória do paciente e o entendimento do profissional dentro do processo do cuidado. Através das entrevistas, no que tange a proposta do IT foram encontradas duas categorias: comunicação e as Redes de Atenção à Saúde (RAS) e o caminhar na rede. Estas estarão sendo desenvolvidas nas seções seguintes.

\section{Comunicação e as redes de atenção}

O acesso aos serviços de saúde pública para o tratamento de doenças respiratórias crônicas (DRC) é ineficaz para o paciente desde a descoberta clara do seu diagnóstico, assim como acaba por ser conflitante para a elaboração e efetiva produção do cuidado por parte dos profissionais de saúde, principalmente os que atuam na Atenção Básica (SOUZA MC, et al., 2017).

A compreensão sobre DRC tem um viés de cunho extremamente popular, que acaba por causar certa negligência na busca por atendimento profissional, fato que contribui para que muitas pessoas iniciam um tratamento quando a doença já está avançada. 
Grande parte dos usuários do SUS são avaliados em Unidades Básicas de Saúde (UBS) ou Unidades de Saúde da Família (USF) até que sejam identificadas as limitações da atenção básica para a continuação do tratamento, momento em que o profissional é orientado a encaminhar o paciente, através de regulação, para um atendimento especializado em doenças respiratórias.

Este encaminhamento é efetivo através das Redes de Atenção à Saúde (RAS), modelo organizacional preconizado pelo Ministério da Saúde a fim de integrar e estabelecer uma linha de comunicação direta entre os sistemas de saúde municipais e estaduais com o intuito de garantir os princípios do SUS: universalidade, integralidade e equidade (TASCA R, et al., 2020).

Um dos conflitos pioneiros na análise do itinerário terapêutico é, justamente, a insegurança que o profissional tem quando encaminha o paciente através das RAS; posto que não tenha havido, na prática, a devolução das contrarreferências informando a evolução do paciente para o setor básico, situação que acaba obstruindo a integralidade que deve haver entre os sistemas municipais e estaduais.

A dificuldade em conseguir efetiva integralidade entre os estes setores de esferas distintas, acaba por perpetuar um sentimento de vazio na equipe profissional da atenção primária envolvida no processo do cuidado, como fora relatado por um dos entrevistados.

"[...] Eu nunca vi um caso de um paciente voltar para cá ou voltar a ficha de contrarreferência. Embora existam as Redes de Atenção à Saúde, temos muito esse sentimento de perda, é como se a gente "jogasse o paciente no meio da Rede e ele se perdesse naquele emaranhado" e ficamos pensando onde está o paciente que eu encaminhei e não voltou." (Entrevistado 1)

Nota-se nesta fala que há uma dificuldade organizacional dos serviços de saúde, que acaba refletindo no cuidado fragilizado que acomete os pacientes com DRC. É inevitável a importância da atenção secundária, que denotam as demandas de média complexidade, com atendimento especializados que são extremamente necessários para o claro diagnóstico de algumas DRCs, mas se não houver clara comunicação com a atenção primária, o tratamento do doente acaba sendo concedido de forma fragmentada.

A precaução em estabelecer essa troca de informações, preconizadas através das RAS, salienta a importância da atenção básica por ser a porta de entrada para o tratamento das DRCs devendo ter uma assistência abrangente destinada a este serviço, sendo essencial para o entendimento do itinerário terapêutico do doente, o que era observado no campo, durante a pesquisa o quanto as pessoas não conheciam/entendiam a rede.

Portanto, uma das características essenciais das RAS é, justamente, formar relações horizontais entre os diferentes pontos de atenção a fim de aperfeiçoar o serviço de saúde e contemplar todos os usuários com um trabalho interprofissional de excelência (Lima DKRR e Guimarães J, 2019).

Entretanto, uma das questões mais relevantes expostas durante a pesquisa, através da perspectiva dos profissionais de saúde que atuam na atenção primária, é que na prática não tem existido uma comunicação segura entre os diferentes níveis de atenção. O esclarecimento ineficaz entre o corpo profissional da atenção primária e secundária prejudica diretamente o processo de atenção à saúde de pessoas com DRC, fragmentando o cuidado e estendendo seu itinerário terapêutico (SALES JCS, et al., 2020).

Uma das consequências do déficit na comunicação entre os níveis de atenção reflete no fato de que as informações sobre o funcionamento das RAS não são passadas para os usuários porque nem mesmo os profissionais conseguem compreender sendo que as redes de atenção fazem parte de um projeto da saúde pública brasileira na tentativa de aperfeiçoar os serviços, melhorar a qualidade sanitária, deixar os usuários satisfeitos e possibilitar uma máxima redução em custos dos sistemas de atenção à saúde (MENDES EV, 2010).

Porquanto, a negligente comunicação sobre como trabalhar nas redes acaba dificultando uma metodologia existente para intervir beneficamente no processo do cuidado desobstruindo as barreiras de uma terapêutica fracionada. Tais lacunas de silêncio entre os protagonistas do cuidado acabam caracterizando a rede com um aspecto (des) funcional fragmentado. 
É crucial salientar que a organização das RAS compreende que o desempenho em equipe é baseado na mútua influência que distintos profissionais exercem uns com os outros objetivando desenvolver o melhor trabalho dentro das possibilidades cabíveis (LIMA SAV, et al., 2015).

A informação é a ferramenta de subsídio para o acesso do usuário ao tratamento e a omissão desta compromete a oferta de uma assistência mais adequada, afirmativa apontada na fala de alguns entrevistados entre pacientes e profissionais de saúde.

\begin{abstract}
"[...] Aí eu fui perguntar para a coordenadora do Distrito onde eu teria acesso porque estava pesquisando na internet, mas eu não achava as Redes de Salvador. Então ela me respondeu "ah, assim... é uma coisa complicada", naquele momento eu tive o sentimento de que nem ela mesmo sabe." (Entrevistado 1).

"[...] Inclusive, tem pessoas onde eu moro que eu disse que ia marcar um pneumologista aqui e disseram: "E lá marca exame?". Eu disse que marcava, porque tem gente que nem sabe." (Entrevistado 9).

"[...] Não, nunca me informaram. Eu fiquei até sabendo pela moça (a farmacêutica) que meu pai quando teve tuberculose tinha direito a medicamento, tinha que ter uma ajuda de custo porque ele ficou sem trabalhar, aí simplesmente ninguém me falou nada." (Entrevistado 4).
\end{abstract}

A desarticulação das práticas de produção de saúde vai contra o princípio de integralidade do SUS, limitando ações de atenção à saúde na prestação do cuidado ao enfermo. Além de não colaborar com o conceito das RAS, impossibilitando uma assistência integral com foco no usuário que facilite o seu deslocamento na rede.

Tal problemática impede outra instância como a promoção de uma relação profissional/usuário mais humanizada, sensível à perspectiva do doente, haja vista que, a produção do cuidado é constituída em caráter subjetivo, podendo ser reconstruída de acordo com os individuais itinerários terapêuticos (CAMUT L, 2017).

A ineficácia da comunicação pode acarretar diversos prejuízos que prolongam o processo terapêutico de pacientes com DRC, sendo importante entender que suas consequências vão surgindo em longo prazo acometendo um sentimento de impotência entre os sujeitos envolvidos (profissionais, pacientes e familiares), por não conseguirem se adaptar com tal falta de integração.

Foi muito comum, ouvir relatos de diversas pessoas o quanto caminharam até chegar ao hospital, principalmente na questão tempo, pois havia casos de pessoas que moravam próximo do hospital, mas que não sabiam do serviço de saúde prestado que atendia a sua necessidade (MACEDO LM e MARTIN STF, 2014).

\title{
O caminhar na "rede"
}

Durante a análise do itinerário terapêutico fora possível detectar que a falta de informação dos funcionários e usuários sobre o funcionamento e especializações das redes de atenção à saúde no que diz respeito ao atendimento de pessoas com DRC, demonstra ser um dos motivos pelo qual o usuário se desloca de forma excessiva em busca da resolubilidade.

Tal situação inviabiliza destreza no atendimento aos enfermos, tornando incompatível a formalização de uma assistência que consiga atender às efetivas necessidades dos usuários. Além de salientar a perspectiva de um sistema de saúde fragmentado que por ineficiência constrange o processo de cuidado do paciente (ROVER MRM, et al., 2016). As afirmativas expostas são consistentes de acordo com esta pesquisa, sendo traduzidas em algumas falas dos entrevistados.

"[...] Foram 4 anos de sofrimento com meu filho, na emergência, de eu não dormir de noite porque tinha que ficar acordada, tinha que elevar o travesseiro, tinha que elevar a cabeceira da cama dele para ele respirar melhor para ele poder conseguir dormir." (Entrevistado 2). 
"[...] Eu andei muito em hospital diferente fazendo tratamento e eles colocavam sempre na ficha "asma crônica". Só que eu nem sei como se diagnostica asma" (Entrevistado 5).

"[...] Não perguntam nada, só escutam a gente. Médico desse tipo, principalmente público, é raro." (Entrevistado 10).

A hegemonia do modelo biomédico, ainda tão focado na patologia, entrelaça os entraves que os dados princípios do SUS tentam desobstruir ao ofertar um serviço que propõe acesso universal à saúde. Tal paradoxo presente no sistema público de saúde consegue afetar de forma negativa e direta o processo do cuidado dos usuários por impossibilitar a compreensão de questões subjetivas e psicológicas que acompanham qualquer doença (SANTOS DS, et al., 2018).

O forte modelo terapêutico mecanicista resiste na saúde pública ainda que sejam elaborados novos métodos de interagir no cuidado, como agrega às RAS, motivo pelo qual é fornecida uma assistência paliativa aos pacientes com DRC permitindo que estes se desloquem muitas vezes durante o itinerário terapêutico (ARRUDA GO, et al., 2018).

Ademais, há a possibilidade destes pacientes não serem bem recepcionados no dia da consulta devido à alta demanda que os profissionais carregam para conseguir contemplar todos os atendimentos do dia. É importante compreender que, quão mais curto for o espaço de tempo do profissional durante a consulta, mais restrito ele pode ficar em conseguir fazer uma ideal análise sobre o IT do paciente.

Outro fator desestimulante para os enfermos verificado nesta pesquisa, são as dificuldades demográficas, econômicas e sociais que grande parte dos pacientes assistidos neste local pesquisado enfrentam durante 0 excessivo deslocamento no processo terapêutico (RESENDE NM, et al., 2017). Segundo relato de alguns entrevistados, tais situações dificultam muito no processo do cuidado.

"[...] Muitas vezes dentro da Atenção Básica a gente não tem a ferramenta necessária para resolver o problema dele, então o que a gente faz é encaminhar." (Entrevistado 1).

"[...] Alguns fazem pelo plano e trazem, mas se for pelo SUS, tem vezes que acaba o grupo (de tabagismo) e não me trazem os exames." (Entrevistado 3).

"[...] Fico em casa. Eu tomo a nebulização, tomo comprimido e melhoro. Porque não tem transporte assim pra gente ir, eu sou de um povoado e o Hospital fica em Monte Santo." (Entrevistado 7).

Nas falas acima, observa-se que há uma dificuldade de acesso para alguns procedimentos, o que se justifica pela burocracia existente e pouco explorada pelos profissionais e pessoas doentes da rede pública, causando insatisfação em muitos usuários e levando-os consequentemente a optar por investir em seguros de saúde particulares a fim de aperfeiçoar o tempo na busca por atendimento e realização de exames, o que concerne com um estudo que demonstra a condição socioeconômica como determinante para o cuidado em saúde (RESENDE NM, et al., 2017).

Ainda que existam dificuldades financeiras na vida dos portadores de DRC entrevistados neste estudo, as tentativas para tentar superar os obstáculos burocráticos e a longa espera por consulta que regem a assistência de saúde pública fazem com que o sistema privado de saúde seja uma opção vantajosa para cessar o deslocamento excessivo que estes enfermos são submetidos durante o itinerário terapêutico.

"[...] Por causa dos meus filhos, foi por eles. Para mim eu me viro, corro aqui e ali. Já no particular tem uma garantia melhor. É um acesso mais fácil.” (Entrevistada 2).

"[...] Marcar clínico, alguma coisa, pedir um check-up. Eu sempre marco no particular." (Entrevistada 9). 
É notável, através das falas dos entrevistados, que há uma falta de direcionamento aos portadores de DRC e seus familiares dentro da Rede para viabilizar a melhor forma de tratamento deste quadro patológico, o que acaba direcionando para o setor privado a resolubilidade da questão. Segundo Carvalho MEL, et al. (2020), a acessibilidade é uma ferramenta que contribui para a produção do cuidado integral, assim como pela capacidade da população para superar estes obstáculos.

O excessivo deslocamento que o usuário carrega como forte característica em seu itinerário terapêutico compreende a diminuta acessibilidade à uma assistência de qualidade, e a comunicação fragmentada entre os diversos níveis de atenção.

A comunicação ineficaz ou a falta desta, dá abertura para um viés que se pode intitular de "Rede Viva", pois o acesso às distintas instituições especializadas em DRC é feita de modo informal entre as pessoas. Os privilégios não institucionais existentes entre usuários do SUS e profissionais atuantes dentro do sistema fazem com que estas pessoas consigam alcançar a consulta desejada sem, necessariamente, enfrentar todo o trâmite burocrático que a Rede estabelece (MAXIMINO VS, et al., 2017).

O somatório de tantas lacunas e problemáticas na produção do cuidado de pessoas com DRC, caracteriza uma atitude recorrente entre alguns pacientes durante o itinerário terapêutico, mesmo aqueles que têm plano de saúde particular: marcar consulta com especialista através de contatos pessoais.

"[...] Não, só que procurasse uma avaliação com o pneumologista. Eu tenho uma filha que trabalha na APAE, tem muitos anos, e com o conhecimento com uma médica que trabalha aqui, ela conseguiu. É muito difícil marcar com esses tipos de médico." (Entrevistada 5).

"[...] Sua consulta foi marcada através de uma amiga aí né?/É.../É parente né?/É." (Entrevistada 8).

Diante das falas dos entrevistados, pode-se afirmar que a comunicação informal atrelada às amizades no setor público de saúde, permite a resolução dos problemas para muitos usuários. Em detrimento de conseguir evitar tantas burocracias das RAS, dentro de um cenário onde o doente portador de DRC necessita de cuidado especializado, mas se depara com pouco direcionamento, essas indicações informais permitem que sejam contemplados com o atendimento mais rápido.

Esta condição cria uma situação nova e diferente nos mecanismos que regem o SUS, caracterizando uma condição diferente no sistema. Consequentemente, a "Rede Viva" acaba sendo mais eficaz para quem tem DRC, pois obtém informação, atendimento, diagnóstico e início de um possível tratamento sem necessitar mais se deslocar tantas vezes pela Rede.

Compreende-se, então, que as barreiras de acesso ao tratamento para pessoas com DRC estão enraizadas na falta de conhecimento e desinformação sobre o funcionamento das redes de atenção à saúde tanto por parte dos profissionais, como dos usuários.

Tal perspectiva abrange um cenário onde há dificuldades para a comunicação e articulação dos diversos níveis de atenção na elaboração do cuidado formalizando uma assistência paliativa que não consegue solucionar as necessidades biológicas e subjetivas dos portadores de doença respiratória crônica (HADAD ACAC e JORGE AO, 2018).

\section{CONCLUSÃO}

Com fundamento nos dados do presente estudo, identificou-se o Itinerário Terapêutico (IT) percorrido por pessoas com doenças respiratórias crônicas (DRC). O estudo evidenciou que grande parte dos pacientes e profissionais de saúde são desinformados sobre o funcionamento das Redes de Atenção à Saúde (RAS), caracterizando um processo de cuidado ainda muito fracionado entre os diversos níveis de atenção, conduta adversa ao que rege os princípios das RAS. Pode-se ainda afirmar que, as doenças do aparelho respiratório não acontecem isoladas, e comungam de diversos fatores que fogem da compreensão biologicista. 


\section{REFERÊNCIAS}

1. ARRUDA G, et al. Percepções da família acerca do cuidado ao homem com alguma condição crônica. Ciência, cuidado e saúde, 2018; 17(1): 1-10.

2. BACKES DS, et al. O Sistema Único de Saúde idealizado versus o realizado: contribuições da Enfermagem. Revista Latino-Americana de Enfermagem, 2014; 22(6): 1026-1033.

3. BRASIL. Ministério da Saúde. Sistema de Informações Hospitalares - SIH/SUS. 2018.

4. CARNUT L. integralidade e atenção primária: articulação essencial para refletir sobre o setor saúde no BRASIL. Saúde em Debate, 2017; 41(115): 1177-1186.

5. CARVALHO MEL, et al. A acessibilidade nos serviços de saúde sob a perspectiva da pessoa com deficiência, Recife - PE. REAS, 2020; 12(1): 1-9.

6. COQUEIRO JM, et al. Itinerários Terapêuticos de Sujeitos com Diabetes Mellitus: Linhas Fortes e Fracas. Rev. Enferm. UFPE, 2018; 12(6): 1554-62.

7. HADAD ACAC e JORGE AO. Continuidade do cuidado em rede e os movimentos de redes vivas nas trajetórias do usuário-guia. Saúde em Debate, 2018; 42: 198-210.

8. LIMA DKRR e GUIMARÃES J. Articulação da Rede de Atenção Psicossocial e continuidade do cuidado em território: problematizando possíveis relações. Physis: Revista de Saúde Coletiva, 2019; 29(3): 1-20.

9. LIMA SAV, et al. Elementos que influenciam o acesso à atenção primária na perspectiva dos profissionais e dos usuários de uma rede de serviços de saúde do Recife. Physis Revista de Saúde Coletiva, 2015; 25(2): 635-656.

10. MACEDO LM e MARTIN STF. Interdependência entre os níveis de atenção do Sistema Único de Saúde (SUS): significado de integralidade apresentado por trabalhadores da Atenção Primária. Interface - Comunicação, Saúde, Educação (Botucatu), 2014; 18(51): 647-660.

11. MAXIMINO VS, et al. Profissionais como produtores de redes: tramas e conexões no cuidado em saúde. Saúde e Sociedade, 2017; 26(2): 435-447.

12. MENDES EV. As redes de atenção à saúde. Rev. Ciências e Saúde Coletiva, 2010; 15(5): 2297-2305.

13. RESENDE NM, et al. Cuidado de pessoas com feridas crônicas na Atenção Primária à Saúde. J Manag Prim Health Care, 2017; 8(1): 99-108.

14. ROVER MRM, et al. Da organização do sistema à fragmentação do cuidado: a percepção de usuários, médicos e farmacêuticos sobre o Componente Especializado da Assistência Farmacêutica. Physis: Revista de Saúde Coletiva, 2016; 26(2): 691-711.

15. SALES JCS, et al. Núcleo de Apoio à Saúde da Família na perspectiva de médicos e enfermeiros. Esc. Anna Nery, 2020; 24(1): 1-9.

16. SANTOS DS, et al. Processo de trabalho na Estratégia de Saúde da Família: potencialidades da subjetividade do cuidado para reconfiguração do modelo de atenção. Ciência \& Saúde Coletiva, 2018; 23(3): 861-870.

17. SILVA NEK, et al. Entre fluxos e projetos terapêuticos: revisitando as noções de linha do cuidado em saúde e itinerários terapêuticos. Ciência \& Saúde Coletiva, 2016; 21(3): 843-852.

18. SOUZA MC, et al. Produção de cuidado e a Rede de Atenção à pessoa com Doença Respiratória Crônica: um estudo de revisão. Revista Pesquisa em Fisioterapia. 2017; 7(4): 574-582.

19. TASCA R, et al. Recomendações para o fortalecimento da atenção primária à saúde no Brasil. Rev Panam Salud Publica, 2020; 44:1-8.

20. ZUIM RCB e TRAJMAN A. Itinerário terapêutico de doentes com tuberculose vivendo em situação de rua no Rio de Janeiro. Physis: Revista de Saúde Coletiva, 2018; 28(02): 1-19. 